\title{
Functional Status and Well-Being in People with Myalgic Encephalomyelitis/Chronic Fatigue Syndrome Compared with People with Multiple Sclerosis and Healthy Controls
}

\author{
Caroline C. Kingdon $^{1} \cdot$ Erinna W. Bowman ${ }^{1} \cdot$ Hayley Curran ${ }^{1} \cdot$ Luis Nacul $^{1}$. \\ Eliana M. Lacerda ${ }^{1}$
}

Published online: 13 March 2018

(C) The Author(s) 2018. This article is an open access publication

\begin{abstract}
Background People with myalgic encephalomyelitis/ chronic fatigue syndrome (ME/CFS) continue to struggle to have their condition recognised as disabling in the face of public and professional prejudice and discrimination.

Objective The aim of this study was to compare the functional status and well-being of people with well-characterised ME/CFS with people with multiple sclerosis (PWMS), as well as healthy controls (HCs).

Methods In this cross-sectional study, we used data collected as part of the UK ME/CFS Biobank to compare actual participant scores from the Medical Outcomes Survey Short Form-36 v2 ${ }^{\mathrm{TM}}$ (SF-36v2 $2^{\mathrm{TM}}$ ) between groups, as a proxy for impact of disability, and from a bespoke questionnaire seeking data on employment and income.

Results People with ME/CFS scored significantly lower than PWMS or HCs in almost all SF-36v2 $2^{\mathrm{TM}}$ areas. Prominent were lower scores for people with ME/CFS in the Physical Component Summary and Role Physical and Social Function domains, while the smallest differences were seen in the Mental Health domain. Responses to the bespoke questionnaire indicated that people with ME/CFS in this study work fewer hours and have lower incomes compared with people in the other two groups.
\end{abstract}

Electronic supplementary material The online version of this article (https://doi.org/10.1007/s41669-018-0071-6) contains supplementary material, which is available to authorized users.

Caroline C. Kingdon

caroline.kingdon@1shtm.ac.uk

1 International Centre for Evidence in Disability, Department of Clinical Research, Faculty of Infectious and Tropical Diseases, London School of Hygiene and Tropical Medicine, Keppel Street, London WC1E 7HT, UK
Conclusions Using SF-36v2 $2^{\mathrm{TM}}$ scores as a proxy, people with ME/CFS were measurably more disabled than PWMS or HCs in this study population. Furthermore, employment and income data are consistent with loss of functional status. These findings should encourage the health community to recognise the disabling effects of ME/CFS, to advocate for the needs of people with ME/CFS, and to investigate strategies to address the cost of the disease to both individuals and society.

\section{Key Points for Decision Makers}

This study suggests that at a group level people with myalgic encephalomyelitis/chronic fatigue syndrome (ME/CFS) are more disabled than people affected by multiple sclerosis (MS), as measured by the SF$36 \mathrm{v} 2^{\mathrm{TM}}$.

$\mathrm{ME} / \mathrm{CFS}$ is associated with a reduction in time spent at work and lower income, compared with people affected by MS.

Further efforts should be made to identify and address the impact of ME/CFS to individuals and society.

\section{Introduction}

While the majority of people with disabilities seek to be recognised first as individuals, independent of their disability [1], people with myalgic encephalomyelitis/chronic 
fatigue syndrome (ME/CFS) continue to campaign to have their disease recognised as disabling [2]. However, research into disability in ME/CFS has been hampered by a lack of consensus, both around the definition of the disease and appropriate measurement tools [3, 4].

ME/CFS is a poorly understood disease. Its measured population prevalence varies between 0.1 and $2.5 \%$ [5-8] according to the definition used, the place and context in which the definition is used, and user perceptions. There is currently no validated test available that can distinguish people with the disease from those without, and, in the absence of a diagnostic marker [9], definitions continue to challenge the medical and research communities [10]. The definitions most widely used by researchers are the US Centers for Disease Control and Prevention (CDC) 1994 case definition [11], the Canadian Consensus Criteria (CCC) [12] and the International Consensus Criteria (ICC) for $\mathrm{ME}$ [13], all of which require the exclusion of other illnesses, except for certain comorbidities.

In adults, ME/CFS is characterised by unexplained persistent or recurrent incapacitating fatigue of more than 6 months' duration accompanied by a range of symptoms that vary within and between individuals. All definitions include a restricted ability to maintain the level of occupational, educational, social and personal activities previously enjoyed [14-16].

The diversity and fluctuating nature of symptoms, the absence of a diagnostic test and the lack of a universally accepted definition have combined to make ME/CFS a disease stigmatised by both the medical community and the public [17-21]. This has likely contributed to limited united efforts by the research community and very restricted funding from government and other mainstream research funders compared with other similarly debilitating illnesses, despite its prevalence and impact [22, 23].

There is little research to quantify health-related quality of life (HRQoL) in people with ME/CFS. Previous research using the Medical Outcomes Survey Short Form-36 v2 $2^{\mathrm{TM}}$ $\left(\mathrm{SF}-36 \mathrm{v} 2^{\mathrm{TM}}\right.$ ) published in 2011 [24] indicated that ME/ CFS impacted the status and well-being of people with well-characterised ME/CFS to a greater extent than 10 other chronic diseases, including cancer. A Danish study published soon thereafter showed that the HRQoL of people in the study with ME/CFS was the lowest of all conditions compared [25], including cancer, diabetes, longand short-term mental illness, stroke, osteoarthritis and rheumatoid arthritis. Both studies relied on general population and disease-specific norms for the comparison groups to show the disabling effects of ME/CFS; a comparison with healthy and diseased groups from the same geographical area from where the cases were provided would likely be more accurate. Furthermore, participants in the Danish study self-reported their diagnosis of ME/CFS, which may have led to selection bias.

In this study, the SF-36v $2^{\mathrm{TM}}$ questionnaire was used to compare functional status and well-being in people with ME/CFS and two matched comparison groups-people with MS (PWMS) and healthy controls (HCs).

\section{Methods}

This cross-sectional study compared SF-36v2 $2^{\mathrm{TM}}$ scores [26] collected from participants in the recently established UK ME/CFS Biobank [27]. The Biobank cohort included people with ME/CFS, PWMS and HCs who had provided data and samples for future research. The data were collected between December 2013 and February 2015 from a population resident in the same geographic area. Participants in the UK ME/CFS Biobank with ME/CFS and MS needed a medically confirmed diagnosis, and people with ME/CFS were required to meet the CDC 1994 [11] or Canadian criteria [12].

PWMS were chosen as a suitable comparison group because (1) both ME and MS are classified as a neurological disease in the World Health Organization's International Classification of Diseases, Tenth Revision (ICD10; G93.3) [28]; and (2) MS (ICD-10; G35) also presents with fluctuating chronic fatigue and a number of other similar symptoms, including orthostatic intolerance and cognitive impairment [29].

\subsection{Recruitment for the UK Myalgic Encephalomyelitis/Chronic Fatigue Syndrome (ME/CFS) Biobank}

Between 2012 and 2015, the UK ME/CFS Biobank collected data and samples from participants in London and East Anglia; details of the UK ME/CFS Biobank protocol have been previously published [27]. Participants were recruited through National Health Service general practices and specialist services with support from the National Institute for Health Research (NIHR) clinical and research networks. Staff from the local NIHR Clinical Research Network (formerly the Primary Care Research Network) approached general practices in Norfolk and Suffolk to seek willingness to recruit participants. Once local ethical approval was in place, participating practices sent letters to registered patients with a diagnosis of ME/CFS, people with a diagnosis of MS, and potential $\mathrm{HCs}$, inviting them to participate in the study. Three HCs were invited for each person with ME/CFS to be recruited. A higher response and recruitment rate was anticipated from people with the disease because people with ME/CFS are underrepresented in research studies and are therefore motivated to 
participate [34]. At the same time, specialty clinics in London, Norfolk and Suffolk approached their patients with ME/CFS and MS. Posters seeking HC volunteers were placed in general practice surgeries and institutes of higher education in the same regions. A full description of recruitment procedures can be found in the paper describing the establishment of the UK ME/CFS Biobank [27].

All participants conformed to the inclusion/exclusion criteria described in Table 1. The study protocol was identical for all participants, regardless of category of recruitment, although questions related to MS and ME/CFS were omitted for HCs.

A clinical researcher assessed all potential Biobank participants at the recruitment stage using a bespoke 'Symptoms Assessment' form to confirm eligibility to participate in the study. All participants donated blood (some of which was used for clinical tests to exclude alternative diagnoses), completed a clinical assessment, and filled out study questionnaires, including the SF$36 \mathrm{v} 2^{\mathrm{TM}}$ and the 'Participant Questionnaire'. Together, these tools enabled the characterisation of participants with $\mathrm{ME} / \mathrm{CFS}$ and MS according to clinical phenotype and disease severity.

\subsection{Sample Size for the Proposed Analysis}

A subsample of 156 individuals (52 with ME/CFS, 52 with MS, and $52 \mathrm{HCs}$ ) was drawn from the Biobank cohort. The participants with ME/CFS were randomly drawn from the list of recruited participants and matched by sex and age group with individuals from the control groups. This sample size was considered sufficient to detect differences in $\mathrm{SF}-36 \mathrm{v} 2^{\mathrm{TM}}$ mean/median scores $\geq 5$ between people with ME/CFS and each of the two comparison subgroups, with a power of $90 \%$ and at a significance level of 0.05 using norm-based scores. We used a conservative 5-point difference in norm-based scores as the minimally important difference (MID), even though the recommended MID varies among the summary measures and each specific domain. For example, an MID of 2-3 points is considered reasonable for the Physical Component Summary (PCS) domain [26], based on clinical trials with patients with peripheral arterial disease. Using a similar approach in different trials, Ware et al. suggested different MIDs for the SF-36v2 ${ }^{\mathrm{TM}}$ scales, ranging from 3.1 to 5.7, the higher MID being recommended for the General Health $(\mathrm{GH})$ domain (5.7), Vitality (VT) domain (5.5) and the Mental Health (MH) domain (5.5). The MIDs recommended for the summary measures were 3.1 (PCS) and 3.8 (Mental Component Summary; MCS) [26].

\subsection{Data Collection}

The SF-36v $2^{\mathrm{TM}}$ is a health survey that uses 36 questions to collect information about functional status and well-being from respondents. These questions evolved from medical outcome studies carried out in the 1980s and 1990s, and were developed with psychometric rigour to ensure that the information captured was reliable [26]. The answers to these unambiguous questions form eight different scales, known as domains. Reports from studies using this instrument evidenced an increase in reliability and validity of scores in a diverse range of populations and settings when compared with other outcome measure instruments

Table 1 UK Myalgic Encephalomyelitis/Chronic Fatigue Syndrome (ME/CFS) Biobank: inclusion and exclusion criteria

\footnotetext{
Inclusion criteria

Age 18-60 years

ME/CFS cases must have a clinical diagnosis using CCC [10] and/or CDC 1994 [23] criteria

MS cases must have a confirmed diagnosis made by an NHS consultant neurologist according to NICE guidelines

Informed consent given

Exclusion criteria corticosteroids), use of antiviral medications, or vaccination infection)

Other severe illnesses

Psychiatric diagnosis (e.g. schizophrenia or severe mood disorder, such as current severe depression)

Pregnant women and those within 12 months' post-partum and/or currently lactating

For ME/CFS and MS cases, other conditions that could explain their chronic fatigue

For healthy controls, current or past fatiguing illness and/or other major morbidity
}

Recent (in the preceding 3 months) use of drugs known to alter immune function (e.g. azathioprine, cyclosporine, methotrexate,

History of acute or chronic infectious diseases such as hepatitis B and C, tuberculosis, HIV (but not herpes virus or other retrovirus

$M E$ myalgic encephalomyelitis, $C F S$ chronic fatigue syndrome, $C C C$ Canadian Consensus Criteria, $C D C$ Centers for Disease Control and Prevention, $M S$ multiple sclerosis, NHS National Health Service, NICE National Institute for Health and Care Excellence, HIV human immunodeficiency virus 
[30]. The SF-36v2 $2^{\mathrm{TM}}$ is recognised as a reliable tool that uses generic health measures that are not age-, disease- or treatment-specific; it has been used and validated across different populations [31]. The version used employs language familiar to people living in the UK [32]. A full report on the development of this research instrument, and a detailed description of its ability to access functional status and well-being, has been published elsewhere [26].

The SF-36v2 ${ }^{\mathrm{TM}}$ considers physical and mental functions resulting in eight distinct domains, which are summarised into the PCS and the MCS [26]. The PCS comprises four domains and is used to profile functional health: Physical Function (PF) measures the ability to undertake everyday activities; Role Physical (RP) measures limitations in the ability to work; Bodily Pain (BP) measures the impact of pain on activity; and GH looks at the general health perceptions of the respondent. Low scores in the PCS indicate reduced functional status.

The four domains making up the MCS are measures of VT, with questions about energy and tiredness; Social Function (SF); MH, which asks about psychological wellbeing, anxiety and depression; and Role Emotional (RE), which considers role limitations due to emotional problems. Since the VT domain is included in the MCS, the physical symptoms of ME/CFS and MS, especially fatigue, are likely to reduce that score, which should be borne in mind when interpreting results.

In this study, the SF-36v2 $2^{\mathrm{TM}}$ instrument was used to compare disability (measuring functional status and wellbeing) and served to assess HRQoL. In addition to the SF$36 \mathrm{v} 2^{\mathrm{TM}}$ questionnaire, all Biobank participants also completed the Participant Questionnaire, which was developed by the UK ME/CFS Biobank team to capture information on socioeconomic, demographic, clinical and family history, and which was piloted prior to its use. Most of its questions about symptoms were taken from validated questionnaires [27, 33-35]. Examples of the questions on employment and income used in the Participant Questionnaire can be seen in Online Appendix 1.

The time lag between disease onset and the response to the questionnaires varied between participants. For the analyses carried out for this study, we used the resultant demographic (sex and age), clinical (functional status and well-being), and socioeconomic (education, accommodation, employment, and income) data. Income data refers to income at the time of recruitment and, when relevant, to 6 months before the disease showed symptoms.

\subsection{Data Analysis}

Answers to the SF-36v2 ${ }^{\mathrm{TM}}$ questions were scored in health domains grouped into the PCS and the MCS using the SF Health Outcomes ${ }^{\mathrm{TM}}$ Scoring Software 4.5 [36].
Although actual scores for individual questions may vary within the general population, these average scores become 50 out of a possible 100 across all questions when they are norm-based with an $\operatorname{SD}(\sigma)$ of 10 [26]. Therefore, norm-based scores compare results with others who have completed the SF-36v2 $2^{\mathrm{TM}}$ rather than against fixed criteria.

Data were analysed using Stata 14.0 software [37]. Bivariate analyses were used to compare people with ME against PWMS or HCs using the Chi-square test and Chisquare for trend to assess the association between categorical variables; the $t$ test or Wilcoxon rank-sum test were used for continuous variables.

In addition to using the SF-36v2 $2^{\mathrm{TM}}$ scores, we also looked at the effect of ME/CFS on some socioeconomic variables, which could also impact HRQoL. For these analyses, we used the variables 'current income', 'employment', 'hours of work' and 'benefits', using conditional logistic regression as recommended for paired design [38].

\section{Results}

\subsection{Description of the Study Population}

The study population came from South East England, was predominantly female $(73 \%)$, of working age (mean age was 49.4 years), and most had completed higher education. Social demographic characteristics are shown in Table 2.

The SF-36v2 ${ }^{\mathrm{TM}}$ responses were analysed from the subsample of participants matched by age and sex, i.e. 52 people with ME/CFS (as per study), 52 PWMS, and 52 $\mathrm{HCs}$, and most participants came from greater London or East Anglia. Of the 52 participants with ME/CFS, 75\% had mild-moderate symptoms and $25 \%$ had severe symptoms, reflecting an intentional recruitment ratio. Of those with MS who responded to the question $(n=42), 71 \%$ had remitting-relapsing MS and $29 \%$ had primary- or secondary-progressive MS.

\section{2 $\mathrm{SF}-36 \mathrm{v} 2^{\mathrm{TM}}$ Comparison Scores}

The SF-36v2 ${ }^{\mathrm{TM}}$ median scores, compared by groups, are shown in Fig. 1, which presents median scores for individual scales as well as the PCS and MCS. This figure is a spider chart, where axes radiate outwards from a central point (0), with each axis representing an SF-36v2 $2^{\mathrm{TM}}$ scale or component summary. The median scores found for the scales and component summaries in each group were plotted on the axes in the spider chart. By joining the points on the axes by group, the resulting polygons clearly displayed the differences in scores from people with ME/CFS 
Table 2 Social demographics of the study sample

\begin{tabular}{|c|c|c|c|c|c|}
\hline Characteristics & $\begin{array}{l}\text { People with ME/CFS } \\
{[n=52]}\end{array}$ & $\begin{array}{l}\text { Healthy controls } \\
{[n=52]}\end{array}$ & $\begin{array}{l}P \text { value }{ }^{\mathrm{a}}[\mathrm{ME} / \\
\mathrm{HCs}]\end{array}$ & $\begin{array}{l}\text { People with MS } \\
{[n=52]}\end{array}$ & $\begin{array}{l}P \text { value }{ }^{\mathrm{a}}[\mathrm{ME} / \\
\mathrm{MS}]\end{array}$ \\
\hline Mean age, years & 49.3 & 49.4 & 0.76 & 49.4 & 0.85 \\
\hline Female & $38(73)$ & $38(73)$ & - & $38(73)$ & - \\
\hline Education & {$[n=52]$} & {$[n=52]$} & 0.88 & {$[n=51]$} & 0.33 \\
\hline Up to $\mathrm{GCSE}^{\mathrm{b}}$ & $17(32.7)$ & $14(26.9)$ & - & $18(35.3)$ & - \\
\hline $\begin{array}{l}\text { Completed school and began higher } \\
\text { education }\end{array}$ & $8(15.4)$ & $10(19.2)$ & - & $9(17.7)$ & - \\
\hline Completed higher education & 15 (28.9) & $17(32.7)$ & - & $19(37.3)$ & - \\
\hline Postgraduate education & $12(23.1)$ & $11(21.1)$ & - & $5(9.8)$ & - \\
\hline Accommodation & & & 0.2 & & 0.6 \\
\hline Owned or mortgaged & $38(73.1)$ & $44(84.7)$ & - & $38(73.1)$ & - \\
\hline Rented & $9(17.3)$ & $8(15.4)$ & - & $13(25)$ & - \\
\hline Other arrangements & $5(9.6)$ & - & - & $1(1.9)$ & - \\
\hline
\end{tabular}

Data are expressed as $n(\%)$

$M E$ myalgic encephalomyelitis, CFS chronic fatigue syndrome, HCs healthy controls, $M S$ multiple sclerosis, GCSE General Certificate of Secondary Education

${ }^{a}$ Chi-square test or Chi-square for trend, or the Wilcoxon rank-sum test

${ }^{\mathrm{b}}$ Secondary school examinations typically taken at the age of 16 years in the UK

(smaller polygon reflects lower scores), PWMS and HCs (higher scores); apart from RE, these differences were all statistically significant. Median SF-36v2 ${ }^{\mathrm{TM}}$ scores from people with ME/CFS were consistently below those for both HCs $(p<0.001$ for all domains and summary measures) and PWMS ( $p<0.03$ for MH scores, and $p<0.001$ for the remaining domains, except for RE, which was 0.12). SF scores, at 27 and 57 for people with ME/CFS and HCs, respectively, showed the largest difference $(p<0.001)$.

\subsection{Socioeconomic Impact}

\subsubsection{Work and Study Hours}

Six months before illness onset, the profiles of hours of study and work (including unpaid and voluntary work, and looking after the home) for people with ME/CFS and PWMS were similar, suggesting that prior to disease onset people in both disease groups were equally active in work and study. Moreover, the pre-illness profiles were similar to those of the HCs.

At recruitment (Fig. 2), 74\% of people with ME/CFS were working and/or studying for 16 or fewer hours/week compared with $26 \%$ of PWMS and $12 \%$ of HCs. Comparing people with ME/CFS pre- and post disease onset, we found that the number of those spending fewer than $17 \mathrm{~h}$ per week at work or study increased more than fourfold (from $17.6 \%$ to $76.4 \%$ ). In this study population, almost
$60 \%$ of HCs reported working and/or studying more than $40 \mathrm{~h} /$ week, compared with $10 \%$ of people with ME/CFS $(p<0.05)$.

\subsubsection{Employment}

Disease onset had a greater impact on employment among people with ME/CFS than among PWMS (Fig. 3), in both working status and number of hours worked. While over $89 \%(n=45)$ of people with ME/CFS had been employed before disease onset, this reduced to $35 \%(n=18)$ after illness onset, which compares with 93\% of PWMS before $(n=48)$ and $60 \%$ of PWMS $(n=52)$ after disease onset.

Only $13 \%$ of people with ME/CFS and $37 \%$ of PWMS worked full time at the time of recruitment, with $52 \%$ of people with ME/CFS and 27\% of PWMS self-identifying as 'sick or disabled', although the differences between the groups did not reach statistical significance $(p=0.07)$.

\subsubsection{Income}

The income of people with ME/CFS and PWMS prior to disease onset followed a normal distribution and there was no statistical difference between the two categories $(p=0.63)$. Free-text data showed that all people with ME/ CFS and PWMS who had not been receiving any income were in full-time education.

The annual income of people with ME/CFS after disease onset was lower than it had been 6 months before disease 


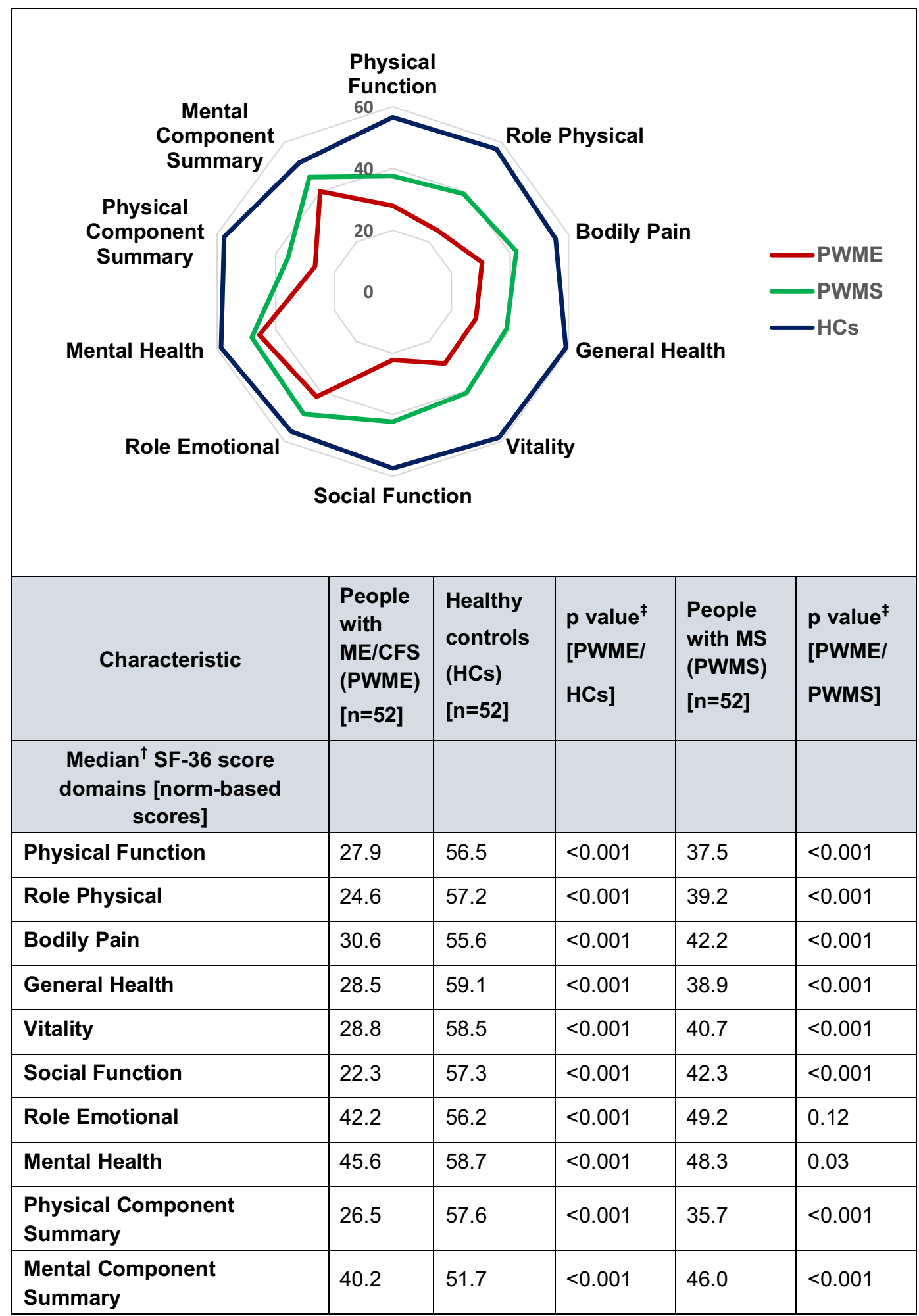

† Because values did not follow a normal distribution, median rather than mean scores are presented; lower scores reflect increased disability.

‡ Wilcoxon rank-sum test. Differences were considered significant when $p$ values were less than 0.05 .

Fig. 1 Median SF-36v2 ${ }^{\mathrm{TM}}$ scores for people with ME/CFS, people with MS, and healthy controls. ME myalgic encephalomyelitis, $C F S$ chronic fatigue syndrome, $M S$ multiple sclerosis 
Fig. 2 Weekly hours of work and study for healthy controls, people with ME/CFS, and people with MS, at recruitment (post-disease onset). $M E$ myalgic encephalomyelitis, $C F S$ chronic fatigue syndrome, $M S$ multiple sclerosis

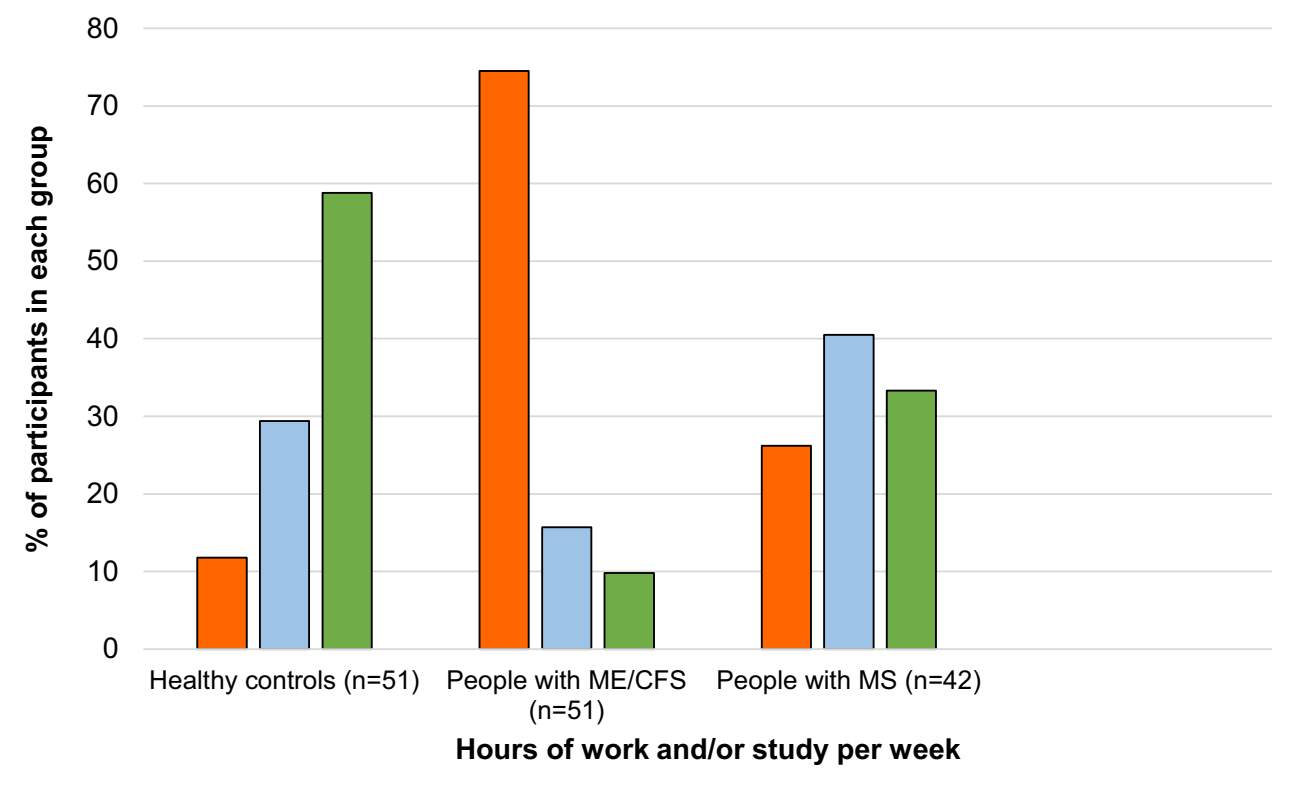

$\square$ Up to 16 hours $\square$ Between 17 and 40 hours $\square$ Over 40 hours

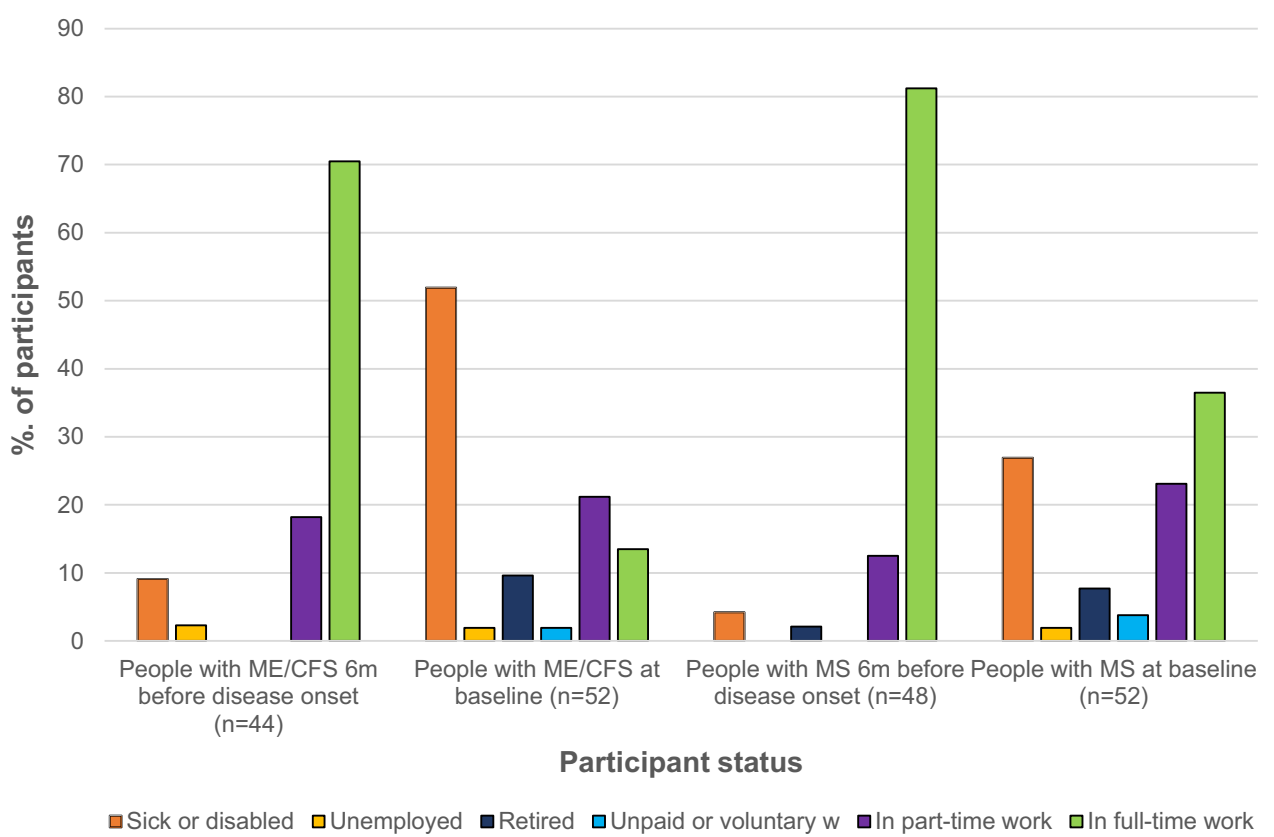

Fig. 3 Employment status of people with ME/CFS and people with MS, at two time points: 6 months before disease onset, and at recruitment. $M E$ myalgic encephalomyelitis, $C F S$ chronic fatigue syndrome, $M S$ multiple sclerosis onset (Fig. 4). The percentage of people with ME/CFS earning more than $£ 30,000$ more than halved following disease onset.

Post disease onset, $83 \%$ of people with ME/CFS earned below $£ 20,000$, compared with 59\% of PWMS and 54\% of HCs (Fig. 5), although these differences were not statistically significant $(p=0.24)$. Some participants did not provide information on income (8\% of people with ME/ CFS and 12\% of PWMS).

Despite the degenerative component that is often part of the natural history of MS, changes in income for PWMS were smaller, at $7.6 \%$, following illness onset. In contrast, the number of people with ME/CFS who earned below $£ 10,000$ increased by $82 \%$.

\subsection{Conditional Logistic Regression Bivariate Analysis}

The bivariate conditional logistic regression analysis results are shown in Table 3, with matching accounting for the potential effect of age group and sex. The odds of people with ME/CFS reporting being unemployed or working part-time were 13.5-fold those of $\mathrm{HCs}(p<0.001)$ and 3.4-fold those of PWMS $(p=0.016)$. These findings 
Fig. 4 Annual income of people with ME/CFS 6 months prior to disease onset and at recruitment. $M E$ myalgic encephalomyelitis, $C F S$ chronic fatigue syndrome, $P W M E$ people with ME/CFS

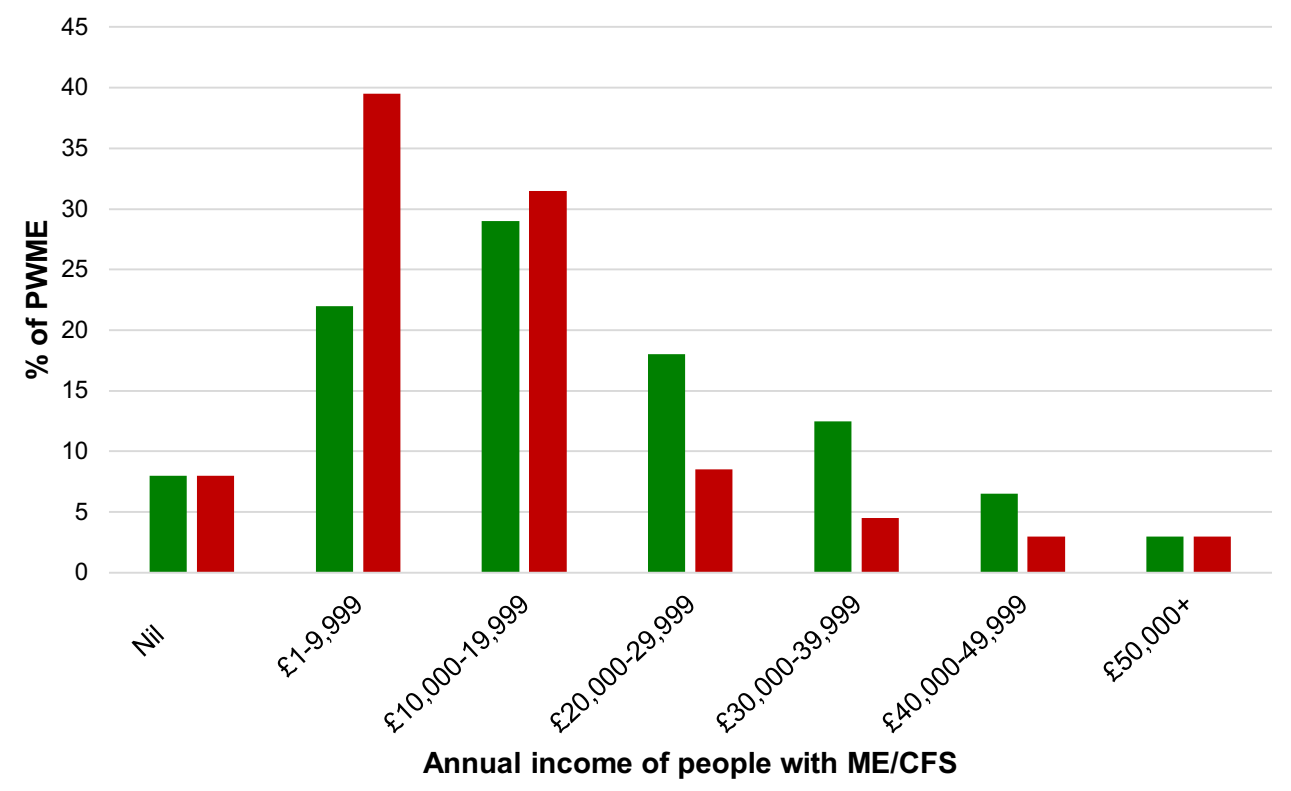

- Percent $6 m$ before onset $(n=49) \quad$ Percent at recruitment $(n=48)$

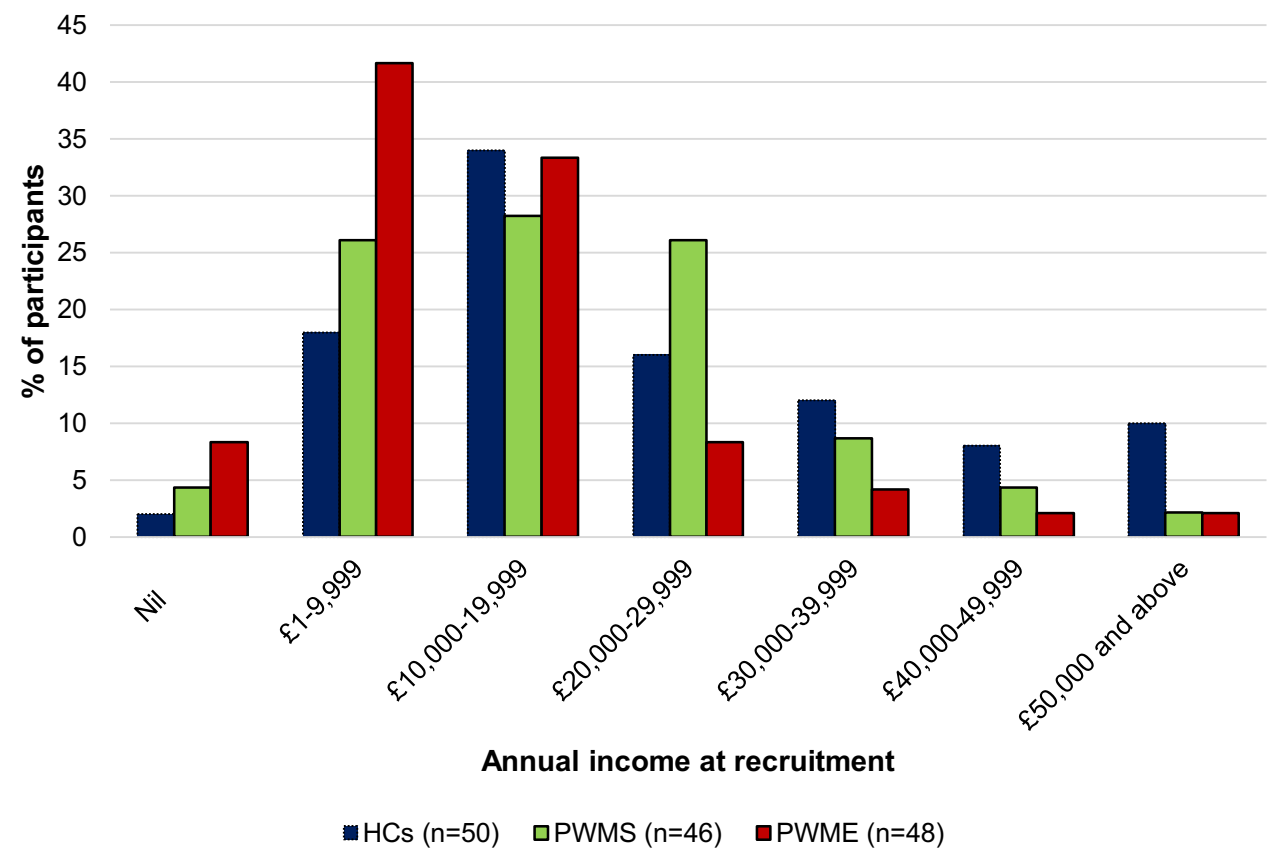

are consistent with the working hours/week from people with ME in relation to HCs and PWMS [odds ratio (OR) $17, p=0.000$; OR 4.6, $p=0.002$, respectively]. Additionally, we found odds of 8.5 for an annual income lower than $£ 19,999$ for people with ME/CFS when compared with HCs $(p=0.004)$, and 3.0 when compared with PWMS ( $p=0.033)$. Furthermore, the odds of people with ME being on benefits was 3.8 of the HCs $(p=0.008)$ and 2.4 those of PWMS (0.048).

\section{Discussion}

Analysis of SF-36v2 ${ }^{\mathrm{TM}}$ data from this study suggests that in almost all areas measured, people with ME/CFS experience greater impact of their disease on life functioning than PWMS. Lower scores in the PCS, RP and SF domains for people with ME/CFS were consistent, corroborating the requirement for a $50 \%$ reduction in activity stipulated in the 2011 ICC [13]. Markedly lower were the PF and SF scores, in line with findings from the National Outcomes 
Table 3 Relationship between ME/CFS and socioeconomic variables (comparing people with ME/CFS with HCs or people with MS) using conditional logistic regression

\begin{tabular}{|c|c|c|c|c|c|}
\hline Independent variable & Dependent variables & OR & $Z$ & $P>(z)$ & $95 \% \mathrm{CI}$ \\
\hline \multirow[t]{4}{*}{ People with ME/CFS (compared with HCs) } & Income $<£ 19,999$ & 8.5 & 2.86 & 0.004 & $1.96-36.79$ \\
\hline & Unemployed or working part-time & 13.5 & 3.55 & $<0.001$ & $3.21-56.77$ \\
\hline & On benefits & 3.8 & 2.66 & 0.008 & $1.42-10.18$ \\
\hline & Working up to $16 \mathrm{~h} /$ week & 17.0 & 3.89 & 0.000 & $4.08-70.76$ \\
\hline \multirow[t]{4}{*}{ People with ME/CFS (compared with people with MS) } & Income $<£ 19,999$ & 3.0 & 2.13 & 0.033 & $1.09-8.25$ \\
\hline & Unemployed or working part-time & 3.4 & 2.41 & 0.016 & $1.25-9.21$ \\
\hline & On benefits & 2.4 & 1.98 & 0.048 & $1.01-5.85$ \\
\hline & Working up to $16 \mathrm{~h}$ /week & 4.6 & 3.09 & 0.002 & $1.75-12.01$ \\
\hline
\end{tabular}

$M E$ myalgic encephalomyelitis, $C F S$ chronic fatigue syndrome, $H C s$ healthy controls, $O R$ odds ratio, $C I$ confidence interval, $M S$ multiple sclerosis

Database in 2010 [39] and supporting the earlier work of Nacul et al. [24].

The study showed statistically significant differences in the number of work and study hours that people with ME/ CFS were able to undertake compared with PWMS and HCs. There were also statistically significant differences in income received by people with ME/CFS, PWMS and HCs. People with ME/CFS were more likely to be unemployed or only able to work part-time than participants in the other two groups. There were noticeable decreases in income in both disease groups, however this was more pronounced in people with ME/CFS.

Study participants with ME/CFS or MS were asked about their income 6 months prior to disease onset and at the time of questionnaire completion; the time between these dates could be as short as 6 months, or as long as many years, depending on disease duration. Such reductions in income actually underestimate actual losses since inflation was not taken into account.

The above associations merit further exploration using longitudinal studies.

\subsection{ME/CFS and the Role of Public Health}

This study provides evidence of the disabling effects of ME/CFS, which negatively impact the HRQoL of people with the disease. Demonstrating that people with ME/CFS can be similarly, or, as suggested in this cohort, more disabled than PWMS, helps to validate ME/CFS as a debilitating illness. Such evidence could energise the public health community to coalesce with health services and various stakeholders to act on behalf of people with ME/CFS by informing policy and aiding advocacy. In turn, this could represent a further argument for increased funding for ME/CFS research and improvements in the planning of care and provision of rehabilitation services for people with ME/CFS. It could also advance the improved education of healthcare workers, many of whom are prejudiced towards ME/CFS and still do not recognise the disease [40].

\subsection{People with ME/CFS: Access to Employment}

These study findings suggest that people with ME/CFS are less able to continue employment after disease onset than PWMS, which may reflect the poorer physical functioning of people with ME/CFS and a lack of appropriate and effective treatments, and which could result from having a disease that is underrecognised and undersupported by employers [41]. Some people with ME/CFS who are able to continue working do so by sacrificing all or much of the social life they would otherwise enjoy [42]. Employers have been shown to be less accommodating to people without a visible disease or infirmity, such as occurs with back pain or depression [43, 44], and their behaviours towards people with ME/CFS are not always positive [41], despite the requirements of the UK Equality Act 2010 [45]. Public health authorities could play a major role in raising awareness of ME/CFS and educating employers around workplace support and modifications. Simple measures such as facilitating rest periods and adjusting light and sound exposure have been shown to be effective [46].

\subsection{The Impact of ME/CFS on Income}

These data demonstrate that in this sample, the income of people with ME/CFS decreased markedly following disease onset and was significantly lower than that of HCs. In this study, raw figures were used, unadjusted for inflation. If inflation were factored into these figures, the decrease in income would be even greater. In the conditional regression analysis, the dependent variables were likely to be 
interacting with each other; a multivariate model analysis would require a larger sample size to account for potential interaction. Repeating these analyses in a larger sample is recommended to further examine the associations between functional status of people with $\mathrm{ME}$ and income. These findings suggest that future analyses on HRQoL, using multivariate models for functional and socioeconomic variables, are worth pursuing.

Loss of employment for people with ME/CFS not only affects the self-worth and economic status of the individual, greatly impacting wellbeing and HRQoL, but also adds to the economic burden on government [47]. Findings from this study are in line with 2012 data from specialist service centres [39], which indicated that slightly more than half of 2000 people with ME/CFS had discontinued employment due to their symptoms. This also aligns with the more than $50 \%$ unemployment found in a systematic literature review of 37 studies in 2004 [9]. Loss of productivity within the UK group was estimated then at over $£ 22,000$ per patient per annum [39]. A recent report estimated the total cost to the UK economy at a minimum of $£ 3.3$ billion anually [48]. Similar studies in the US have estimated that lost productivity for people with ME/CFS could be from $\$ 9$ billion to $\$ 37$ billion per annum [49]. While investment may be essential to support employment opportunities, the decreased need for benefits and increased tax revenue from an employed population may help to address such costs [50], and robust economic analysis should inform public health thinking.

\subsection{Study Limitations}

It is difficult to generalise these results. Data were collected from mainly White participants in South East England, therefore the results may not be applicable to minority populations, although there is no obvious indication that results would differ significantly in other population groups.

All HC SF-36v2 ${ }^{\mathrm{TM}}$ scores were $>50$, i.e. above a general population average, which might be due to selection bias because of the nature of recruitment [51]. People with major illnesses were excluded and HCs agreeing to participate in a study of this kind are likely to be healthier than the average person.

All data were self-reported and some were retrospective, and therefore could have been subject to reporting and recall bias, particularly data on employment and income.

A multivariate analysis to account for potential confounding was not possible due to the likely degree of interaction between the outcome variables and potential confounding variables. Analysis with a larger sample size is planned for the future as the UK ME/CFS Biobank expands.
ME/CFS and MS can fluctuate in severity both day-today and over longer periods. Since symptoms can be quite disabling, participants with ME/CFS in particular were only able to participate during a time in which they were feeling able to do so, and many appointments had to be rescheduled. This presents a challenge for a cross-sectional analysis such as this since the answers provided may not be fully representative of the experience of participants with ME/CFS on a typical day insofar as the results may underrepresent the impact and/or severity of ME/CFS symptoms.

\section{Conclusions}

This study provides evidence for the reality of disability for many people with ME/CFS and the disease's effect on HRQoL and wellbeing. In this study population, the loss of functional status, as a proxy for disability, was greater in people with ME/CFS than PWMS. The disease conspicuously reduced SF in people with ME/CFS, and HRQoL may have been impacted by the subsequent social isolation [52]. More people with ME/CFS than PWMS lost their jobs after becoming ill, and the income of people with ME/CFS decreased markedly more than for PWMS. Unemployment costs are borne by both the individual and society. The prevalence of ME/CFS, the evident reduction in HRQoL of those affected, and the economic cost of the illness to society should be powerful motivators for policy makers to encourage better funding of research to discover the cause of, and establish effective treatments for, this disease [53].

Validating these findings will require further research with a larger sample size and in other population-based samples.

Author Contributions Caroline Kingdon conceptualised the study, assessed research participants, analysed the data and drafted the research paper. Eliana Lacerda participated in the study design, guided the data analysis and interpretation of results, and helped with the manuscript write-up; she also contributed to the authorship of the paper. Erinna Bowman contributed to the authorship and critical review of the paper. Hayley Curran contributed to the authorship and critical review of the paper. Luis Nacul provided input to data interpretation and write-up, and helped to critically review and approve the final version for publication.

Data Availability Statement Participants gave permission for the data to be made available, in a de-identified format, to researchers with ethically approved projects. The data are therefore available from the authors on reasonable request and following peer review of any application for data use (http://cureme.lshtm.ac.uk/researchers/ accessing-the-biobank/ or http://cureme.lshtm.ac.uk/researchers/ protocols-application). 


\section{Compliance with Ethical Standards}

Funding The UK ME/CFS Biobank was established with a joint grant from the charities Action for ME, the ME Association, and ME Research UK, as well as a private donor. Research reported in this publication was supported by the above-named charities and the National Institute of Allergy and Infectious Diseases (NIAID) of the National Institutes of Health (NIH) under Award Number R01AI103629. The content is solely the responsibility of the authors and does not necessarily represent the official views of the NIH.

Conflicts of Interest Caroline Kingdon, Erinna Bowman, Hayley Curran, Luis Nacul, and Eliana Lacerda declare that they have no conflicts of interest.

Human Participants All procedures performed in studies involving human participants were in accordance with the ethical standards of the institutional and/or national research committee and with the 1964 Helsinki declaration and its later amendments or comparable ethical standards.

Ethical Approval The UK ME/CFS Biobank received ethical approval from the London School of Hygiene \& Tropical Medicine (LSHTM) Ethics Committee (ref. 6123), the National Research Ethics Service (NRES) London-Bloomsbury Research Ethics Committee (REC; ref. 11/LO/1760, IRAS ID: 77765), and the NHS Research Governance and Developments Offices (R\&D), which oversee the recruitment of research participants from government health services.

Informed Consent Written informed consent was obtained from all individual participants included in the study. Participants were encouraged to ask questions and were free to withdraw at any time.

Open Access This article is distributed under the terms of the Creative Commons Attribution-NonCommercial 4.0 International License (http://creativecommons.org/licenses/by-nc/4.0/), which permits any noncommercial use, distribution, and reproduction in any medium, provided you give appropriate credit to the original author(s) and the source, provide a link to the Creative Commons license, and indicate if changes were made.

\section{References}

1. Olive M. The politics of disablement: a sociological approach critical texts in social work and the welfare state. Basingstoke: Macmillan and St Martin's Press; 1990.

2. ME Association. ESA - changes to the Working Capability Assessment descriptors. 2011. http://www.meassociation.org.uk/ 2011/02/esa---changes-to-the-working-capability-assessmentdescriptors/. Accessed 28 Feb 2018.

3. Strassheim V, et al. What is known about severe and very severe chronic fatigue syndrome? A scoping review. Fatigue Biomed Health Behav. 2017;5(3):167-83.

4. Cella M, Sharpe M, Chalder T. Measuring disability in patients with chronic fatigue syndrome: reliability and validity of the Work and Social Adjustment Scale. J Psychosom Res. 2011;71(3):124-8.

5. Dinos S, et al. A systematic review of chronic fatigue, its syndromes and ethnicity: prevalence, severity, co-morbidity and coping. Int J Epidemiol. 2009;38:1554-70.

6. Prins J, van der Meer J, Bleijenberg G. Chronic fatigue syndrome. Lancet. 2006;367:346-55.
7. Bakken I, et al. Two age peaks in the incidence of chronic fatigue syndrome/myalgic encephalomyelitis: a population-based registry study from Norway 2008 inverted question mark 2012. BMC Med. 2014;12(1):167.

8. Nacul LC, et al. Prevalence of myalgic encephalomyelitis/chronic fatigue syndrome (ME/CFS) in three regions of England: a repeated cross-sectional study in primary care. BMC Med. 2011;9(1):91.

9. Ross S, et al. Disability and chronic fatigue syndrome. Arch Intern Med. 2004;164:1098-105.

10. Nacul LK, Kingdon CC, Bowman EW, Curran H, Lacerda EM. Differing case definitions point to the need for an accurate diagnosis of myalgic encephalomyelitis/chronic fatigue syndrome. Fatigue Biomed Health Behav. 2017;5(1):1-4.

11. Fukuda K, et al. The chronic fatigue syndrome: a comprehensive approach to its definition and study. International Chronic Fatigue Syndrome Study Group. Ann Intern Med. 1994;121(12):953-9.

12. Carruthers, et al. Myalgic encephalomyelitis/chronic fatigue syndrome: clinical working case definition, diagnostic and treatment protocols. J Chronic Fatigue Syndr. 2003;11(1):7-115.

13. Carruthers BM, et al. Myalgic encephalomyelitis: international Consensus Criteria. J Intern Med. 2011;270(4):327-38.

14. Baker R, Shaw E. Diagnosis and management of chronic fatigue syndrome or myalgic encephalomyelitis (or encephalopathy): summary of NICE guidance. BMJ. 2007;335:446-8.

15. Carruthers B, et al. Myalgic encephalomyelitis/chronic fatigue syndrome: clinical working case definition, diagnostic and treatment protocols. J Chronic Fatigue Syndr. 2003;11(7):115.

16. Fukuda K, et al. The chronic fatigue syndrome: a comprehensive approach to its definition and study. International Chronic Fatigue Syndrome Study Group. Ann Intern Med. 1994;121:953-9.

17. Anderson V, et al. A review and meta-synthesis of qualitative studies on myalgic encephalomyelitis/chronic fatigue syndrome. Patient Educ Couns. 2012;86(2):147-55.

18. Åsbring P, Närvänen A-L. Women's experiences of stigma in relation to chronic fatigue syndrome and fibromyalgia. Qual Health Res. 2002;12(2):148-60.

19. Blease C, Carel H, Geraghty K. Epistemic injustice in healthcare encounters: evidence from chronic fatigue syndrome. J Med Ethics. 2017;43:549-57.

20. Drachler Mde L, et al. The expressed needs of people with chronic fatigue syndrome/myalgic encephalomyelitis: a systematic review. BMC Public Health. 2009;9:458.

21. Thomas MA, Smith AP. Primary healthcare provision and chronic fatigue syndrome: a survey of patients' and general practitioners' beliefs. BMC Fam Pract. 2005;6:49.

22. Horton SM, et al. Chronic fatigue syndrome/myalgic encephalomyelitis (CFS/ME) in adults: a qualitative study of perspectives from professional practice. BMC Fam Pract. 2010;11:89.

23. Green C, et al. Pathways to prevention workshop: advancing the research on myalgic encephalomyelitis/chronic fatigue syndrome. Ann Intern Med. 2015;162(12):860-5.

24. Nacul L, et al. The functional status and well being of people with myalgic encephalomyelitis/chronic fatigue syndrome and their carers. BMC Public Health. 2011;11:402.

25. Falk Hvidberg M, et al. The health-related quality of life for patients with myalgic encephalomyelitis/chronic fatigue syndrome (ME/CFS). PLoS ONE. 2015;10(7):e0132421.

26. Ware JJ, et al. User's manual for the SF-36v2 ${ }^{\mathrm{TM}}$ health survey 2007: Lincoln, RI: QualityMetric Incorporated.

27. Lacerda EM, et al. The UK ME/CFS biobank for biomedical research on myalgic encephalomyelitis/chronic fatigue syndrome (ME/CFS) and multiple sclerosis. Open J Bioresour. 2017;4(1):4. 
28. World Health Organization. International classification of statistical classification of diseases and related health problems-ICD10. Geneva: World Health Organization; 2001.

29. Morris G, Maes M. Myalgic encephalomyelitis/chronic fatigue syndrome and encephalomyelitis disseminata/multiple sclerosis show remarkable levels of similarity in phenomenology and neuroimmune characteristics. BMC Med. 2013;11:205.

30. Ware JJ, Kosinski M, Gandek B. SF- $36^{\circledR}$ Health Survey Manual and Interpretation Guide. Lincoln: QualityMetric Inc; 2005.

31. Myers C, Wilks D. Comparison of Euroqol EQ-5D and SF-36 in patients with chronic fatigue syndrome. Qual Life Res. 1999;8:9-16.

32. Jenkinson $\mathrm{C}$, et al. Assessment of the SF-36 version 2 in the United Kingdom. J Epidemiol Comm Health. 1999;53(1):46-50.

33. Bell D. The doctors' guide to chronic fatigue syndrome: understanding, treating, and living with Cfids. Bell DS, editors. DC Press;1996. pp. 124-125. Boston, MA: Da Capo Press.

34. Johns MW. A new method for measuring daytime sleepiness: the Epworth sleepiness scale. Sleep. 1991;14(6):540-5.

35. Krupp LB, et al. The fatigue severity scale. Application to patients with multiple sclerosis and systemic lupus erythematosus. Arch Neurol. 1989;46(10):1121-3.

36. Ware JEJ, Kosinski M. Interpreting SF-36 Summary Health Measures: a response. Qual Life Res. 2001;10(5):405-13.

37. Stata Corp. Stata statistical software: release 14. College Station: StataCorp LP; 2015.

38. Kirkwood BR, Sterne JAC. Medical statistics. 2nd ed. Oxford: Blackwell Science; 2003.

39. Collin SM, et al. The impact of CFS/ME on employment and productivity in the UK: a cross-sectional study based on the CFS/ ME national outcomes database. BMC Health Serv Res. 2011;11:217.

40. Morris G, Maes M. Myalgic encephalomyelitis/chronic fatigue syndrome and encephalomyelitis disseminata/multiple sclerosis show remarkable levels of similarity in phenomenology and neuroimmune characteristics. BMC Med. 2013;11:205.

41. Shlaes JL, Jason LA, Ferrari JR. The development of the chronic fatigue syndrome attitudes test. A psychometric analysis. Eval Health Prof. 1999;22(4):442-65.

42. Shepherd C. Living with M.E, the chronic post-viral fatigue syndrome. Reading: Vermilion; 1999.
43. Brohan E, Thornicroft G. Stigma and discrimination of mental health problems: workplace implications. Occup Med (Lond). 2010;60(6):414-5.

44. Brunt-Seymour K. How employers' unsympathetic attitudes towards staff absence affect staff productivity. Workplace savings and Benefits 2015. http://www.wsandb.co.uk/wsb/news/ 2424354/how-employers-unsympathetic-attitudes-towards-staffabsence-affect-staff-productivity. Accessed 28 Feb 2018.

45. UK Government. The UK Equality Act 2010. 2010. https://www. hesa.ac.uk/dox/manual_documents/ea-guide.pdf?v=). Accessed 28 Feb 2018.

46. National Institute of Clinical Excellence. Chronic fatigue syndrome/myalgic encephalomyelitis (or encephalopathy): diagnosis and management of CFS/ME in adults and children. NICE guidelines 2014. https://www.nice.org.uk/guidance/cg53. Accessed 17 Jul 2017.

47. Murray CJ, Lopez AD. Evidence-based health policy: lessons from the Global Burden of Disease Study. Science. 1996;274(5288):740-3.

48. Hunter R, James M, Paxman J. Counting the Cost: chronic fatigue syndrome/myalgic encephalomyelitis. Full report 2017. http:// www.theoptimumhealthclinic.com/wpcontent/uploads/2017/09/ Counting-the-Cost.pdf. Accessed 28 Feb 2018.

49. Ross SD, et al. Disability and chronic fatigue syndrome: a focus on function. Arch Intern Med. 2004;164(10):1098-107.

50. Organisation for Economic Co-operation and Development. Tackling the Jobs Crisis: Pathways onto (and off) Disability Benefits: Assessing the Role of Policy and Individual Circumstances. OECD Employment Outlook. OECD; 2009. http://www. oecd.org/employment/emp/43687710.pdf?TSPD_101_R0=d00ce 51dafceec68e8eaf52ee5d42645h0400000000000000005d6d5a92 ffff00000000000000000000000000005a96ce4e00c96b26ba. Accessed 28 Feb 2018.

51. Carneiro I, Howard N. Introduction to Epidemiology. Understanding Public Health. Bungay. 2nd ed. McGraw Hill: Open University Press; 2011.

52. Ubido J, Scott-Samuel A. Loneliness. The prevalence of loneliness, its impact on health and wellbeing and effective interventions that can be used to ameliorate these effects. Rapid Evidence Review Series, number 1, 2014. LPHO Report Series, number 97.

53. Jason L, et al. The Economic impact of ME/CFS: individual and societal costs. Dyn Med. 2008;7:6. 